\title{
Universidad: articulación de la docencia, la investigación y la extensión universitaria
}

\section{University: Articulation of Teaching, Research, and University Extension}

\author{
Alexis Bruno Rodríguez Solís \\ Sede Regional Brunca, Campus Coto \\ Puntarenas, Costa Rica \\ Universidad Nacional \\ alexis.rodriguez.solis@una.cr
}

Recibido: 16/04/2019 Aceptado: 07/08/2019

\begin{abstract}
Resumen. La extensión, junto con la docencia y la investigación, conforman la finalidad académica de la Universidad Nacional, así se puede definir el hacer academia como una vivencia producto de la integración de los tres quehaceres sustantivos. Partiendo de este supuesto, surge la inquietud respecto a cómo lograr que los planteamientos estatutarios, referentes a la importancia de dichos quehaceres, sean puestos en práctica de manera articulada en las distintas instancias académicas y lo más importante cómo lograr que se vean reflejados en la labor de quienes tienen nombramiento con jornada académica. Así, este trabajo tiene como objetivo fundamentar una concepción académica de universidad, que comprende la docencia, la investigación y la extensión con la finalidad de que las instancias universitarias se responsabilicen de su gestión, mediante un accionar cotidiano y planificado a través de la formulación de un programa académico que permita la articulación de esos quehaceres. La articulación de la extensión con la investigación y la docencia debe partir de una clara conceptualización, y la puesta en práctica implicará el compromiso de toda la comunidad universitaria. Para lo cual será necesario en primera instancia una evaluación del modo en que se gestiona la labor académica, lo que lleva a considerar la distribución y los recursos, y posiblemente provocará una reformulación estratégica para lograr una universidad comprometida con la excelencia en la formación, el desarrollo de la investigación y el diálogo con las comunidades.
\end{abstract}

Palabras clave: academia, docencia, extensión, integración, investigación. 


\begin{abstract}
University extension, teaching and research are the academic purposes of the National University of Costa Rica. The integration of these three pillars defines the academy doing as a resulting product of their fulfillment. Based on this assumption, concerns arise on how the statutory considerations referred to the importance of those university tasks are put into practice into the different academic instances in an articulated way. Most importantly, how to make these statutory considerations be reflected in the job of those who have full-time academic job. Hence, the objective of this research consists of supporting an academic conception of university which includes teaching, research and university extension with the purpose of encouraging the university instances to undertake their management through a daily basis action and a planning process by formulating an academic program that allows the articulation of those university tasks. The articulation of extension with research and teaching must be based on a clear conceptualization, and implementation will involve the commitment of the entire university community; for which it will be necessary, as a first instance, an evaluation of how the academic job is managed. That means to consider the distribution of resources, and it is likely to provoke a strategic reformulation to reach a university committed to the excellence of the formation of professionals, the development of research, and the dialogue with communities.
\end{abstract}

Keywords: academy, teaching, extension, integration, research.

\title{
Introducción
}

La Universidad, tal como se concibe, contempla la realización de tres quehaceres sustantivos: la docencia, la investigación y la extensión. La primera dedicada primordialmente, aunque no únicamente, a la transferencia de conocimiento, la segunda a la creación del conocimiento, si bien entraña también la transferencia y la puesta en práctica del mismo, y la tercera a la puesta en práctica del conocimiento, aunque implica también lo que es característico de los otros quehaceres. Las tres actividades se encuentran en un mismo nivel, sin un orden particular y en una relación transitiva, además se vinculan con la sociedad de manera dialógica.

En específico, la extensión en la Universidad Nacional surge desde inicios de la década de los sesenta, y posteriormente en 1984 el Consejo Universitario aprueba las normas específicas que la rigen, propuestas en el I Seminario de Extensión y Difusión Cultural. Históricamente, la extensión ha representado el vínculo entre la universidad y la sociedad, particularmente con los sectores menos favorecidos de esta. 
«La extensión universitaria es la herramienta más enriquecedora de la que dispone la Universidad para lograr múltiples transformaciones porque favorece el desarrollo de las comunidades mediante la creación conjunta de saberes» (Universidad Nacional, 2017b, p. 33). Sin embargo, en la actualidad existe un problema fundamental que dificulta el cumplimiento de esas palabras. El insuficiente desarrollo de la investigación y de la extensión universitarias, situación que se torna más compleja en el caso de la extensión, debido a una deficiente conceptualización, que tiende a una práctica de carácter heterogéneo y a la falta de recursos para su ejecución y asignación de jornadas académicas.

Además, con base en el «Plan de mediano plazo (2017-2021)», la Universidad Nacional enfrenta las siguientes debilidades que afectan la extensión universitaria:

1. En el ámbito académico prevalece una endogamia academicista que obstaculiza el diálogo de saberes y favorece una excesiva fragmentación disciplinaria, que se manifiesta en la organización institucional de facultades y centros, en dinámicas de competencia asimétrica por recursos.

2. Se desarticula la actividad académica y esto provoca la disgregación de la gestión universitaria que induce a duplicar funciones, así como la incomunicación entre las dependencias interuniversitarias.

3. Gran parte de la actividad académica que se realiza en la Universidad se centra en la docencia, con la investigación y la extensión restringidas.

4. En el ámbito académico regional, preocupa visualizar la acción territorial como fragmentada, descoordinada y descontinuada, lo cual propicia acciones que no responden a una estrategia unitaria. Además, en muchas ocasiones la oferta académica no es suficiente, ni articulada; tampoco actualizada, ni completa. (UNA, 2017b, pp. 35-36)

La práctica manifiesta que existe la tendencia a un desarrollo de proyectos y abordajes de extensión, en especial, unidisciplinario, que no necesariamente remite a procesos de diálogo con las diversas comunidades del país o a procesos interdisciplinarios para la atención planificada e impacto social, de acuerdo con necesidades específicas identificadas colectivamente. Además, se han observado en las universidades públicas de Costa Rica, instancias dedicadas enteramente a la docencia, o personal organizado a suerte de exclusividad, en la cual unos hacen la docencia, otros investigan y otros son extensionistas. 
Aún más, cuando se realizan las tres actividades, con la mayor frecuencia no se implican unas con otras, de manera que se realimenten entre sí.

En relación con esto, el «Plan de mediano plazo (2013-2017)» (2017a) estableció como una de sus metas implementar mejoras que permitan superar las asimetrías en la actividad académica, siendo uno de sus indicadores los tiempos completos académicos equivalentes ejecutados por unidad académica y por área académica y según quehacer (docencia, investigación, extensión). Otra de sus metas es generar acciones que articulen el quehacer académico, la construcción colectiva y el desarrollo de las Unidades Académicas, Facultades, Centros y Sedes, siendo uno de sus indicadores la cantidad de acciones que articulan el quehacer académico.

Consecuente con lo anterior, esta ponencia tiene como objetivo fundamentar una concepción académica de universidad que comprende la docencia, la investigación y la extensión, con la finalidad de que las instancias universitarias se responsabilicen de su gestión, mediante un accionar cotidiano y planificado a través de la formulación de un programa académico que permita la real articulación de dichos quehaceres.

\section{Metodología}

El estudio se fundamenta en el análisis cualitativo, abarcando técnicas como la revisión de bibliografía sobre textos relacionados con la extensión universitaria en Costa Rica y la valoración de lo establecido en los distintos instrumentos de organización, planificación y rendición de cuentas de la Universidad Nacional, tales como la normativa, los presupuestos ordinarios (2011 al 2017), los planes anuales operativos operáticos (2012-2017), los planes de mediano plazo (2013-2017 y 2017-2021) y los informes de labores. El análisis pretende encontrar información referente al desarrollo de los quehaceres sustantivos y su articulación.

Además, se considera el número de programas, proyectos y actividades de investigación, extensión, docencia e integrados (proyectos que articulan al menos dos quehaceres académicos) que realizan las instancias académicas, como una variable que permita determinar la intensidad y el desarrollo de dichos quehaceres, así como el grado de consecuencia con una visión tripartita de universidad.

Adicionalmente, se procura realizar un análisis cuantitativo del presupuesto destinado a la docencia, la investigación y la extensión universitarias, y 
URL: http://www.revistas.una.ac.cr/index.php/dialogo/index

CORREO ELECTRÓNICO: universidadendialogo@una.cr

DOI: http://doi.org/10.15359/udre.9-2.1

conocer la forma en que distribuyen las jornadas académicas según el quehacer sustantivo. Con base en esa información se elabora la propuesta referente a la necesidad de promover la formulación de un programa académico en las distintas instancias responsables de la realización de las actividades sustantivas.

\section{Resultados}

Respecto a las jornadas académicas de la Universidad Nacional, se cuenta con información sobre los datos totales de tiempos completos académicos, sin embargo, de manera centralizada no se tienen detalles en relación con la ejecución de esos tiempos por quehacer sustantivo, lo que dificulta evidenciar la carga que representan y conocer las diferencias que puedan existir en la distribución. Si bien los presupuestos ordinarios de la Universidad hacen referencia a la relación laboral, los datos no permiten un análisis certero de cómo se implementan en la práctica. Por otra parte, en este mismo tema el «Plan de mediano plazo (2017-2021)» indica que:

En los últimos años se ha realizado una inversión sustancial al dotar a las unidades académicas de nuevos recursos laborales, para que fortalezcan sus actividades y logren sus objetivos. En el ámbito académico se asignaron 263,75 tiempos completos con el objeto de apoyar aquellas actividades académicas que propicien una actividad sustantiva de la universidad con impacto positivo en la sociedad (2017b, p. 35).

Continuando con la discusión, ese mismo plan indica que «la matrícula de la población estudiantil interesada en ingresar ha experimentado un incremento, así como de quienes realizan prueba y, finalmente, matriculan en la carrera de preferencia» (2017b, p. 31). La figura 1 demuestra el aumento sostenido en la matrícula de estudiantes, lo cual evidencia una labor tendiente al incremento de la docencia universitaria, entendida como la oferta de educación formal disponible.

En lo que respecta a la cantidad de programas, proyectos y actividades de investigación, extensión, docencia e integrados que realizan las instancias académicas, la figura 2 presenta el número desarrollado del 2010 al 2016, demostrando las diferencias existentes entre los quehaceres sustantivos. Destaca la disminución en los proyectos de carácter integrado, que apenas en el año 2016 empiezan a remontar, de igual forma la reducción en investigación; por su parte, la extensión se ha mantenido estable, al igual que la docencia. La única modalidad que muestra un aumento significativo en ese periodo es la llamada gestión académica, que corresponde a proyectos orientados a 
garantizar la pertinencia y la calidad de los procesos académicos, así como la gestión administrativa de las unidades académicas. Por último, cabe indicar que respecto a los llamados integrados la información disponible no permite identificar en la totalidad de los casos la mayor presencia de alguna de las tres actividades sustantivas en su formulación, por lo cual no es conveniente realizar alguna inferencia.

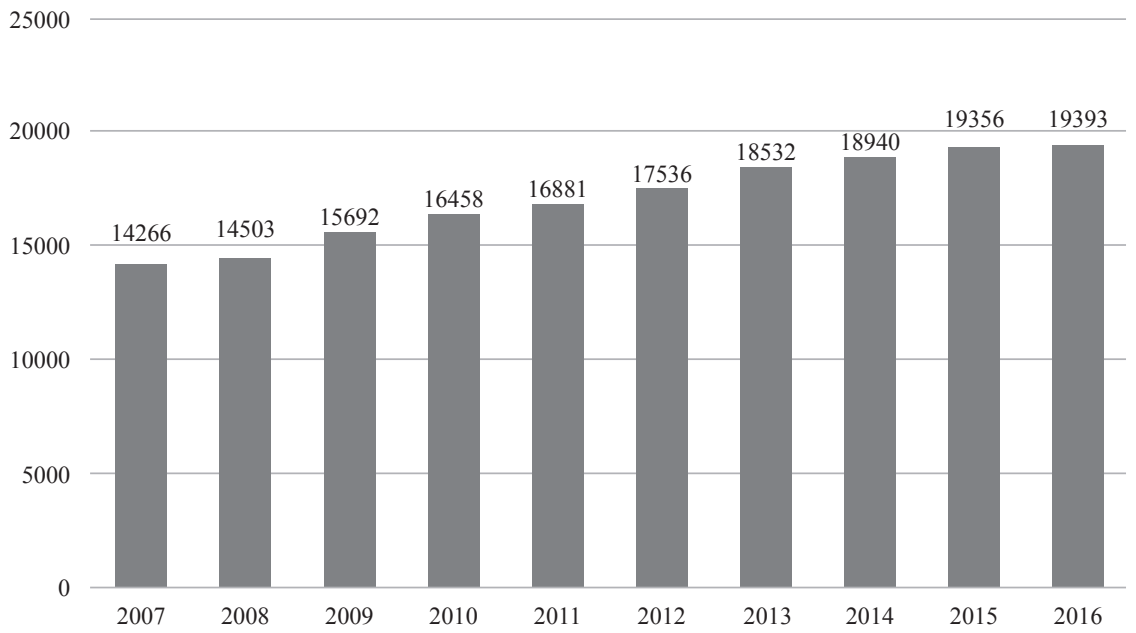

Figura 1. Histórico del total de estudiantes matriculados (2007-2016).

Fuente: Elaboración propia con base en la información disponible en el portal web de transparencia de la Universidad Nacional.

Según información publicada en el portal de transparencia:

La Universidad cuenta con 219 proyectos de investigación activos, que se desarrollan a lo largo de todo el país, 25 revistas internacionales indexadas, y un incremento del $19 \%$ en su labor investigativa entre el 2014 y el 2016. En el ámbito de la Extensión, sólo el año pasado se desarrollaron 64 proyectos, programas y actividades académicas. Por otra parte, la docencia universitaria se levanta como una tercera plataforma fundamental en la proyección del quehacer institucional, con 27 proyectos, programas y actividades académicas para el año 2016 (UNA, 2017c). 
URL: http://www.revistas.una.ac.cr/index.php/dialogo/index

CORREO ELECTRÓNICO: universidadendialogo@una.cr

DOI: http://doi.org/10.15359/udre.9-2.1

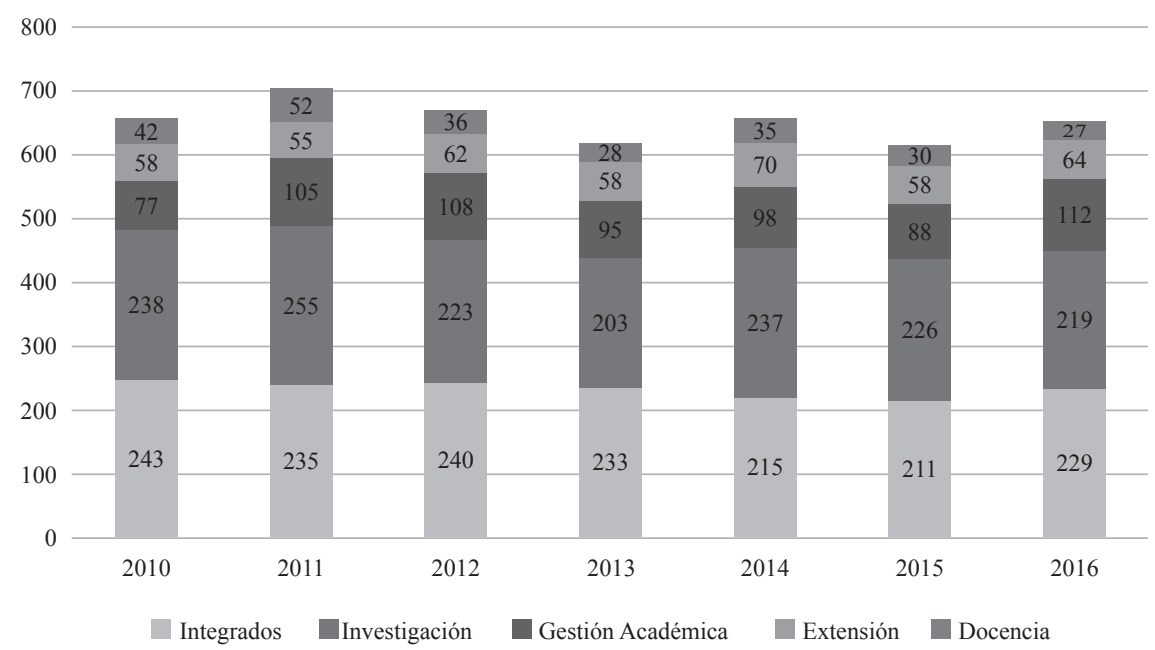

Figura 2. Cantidad de proyectos, programas y actividades académicas por área realizadas del año 2010 al año 2016.

Fuente: Elaboración propia con base en la información disponible en el portal web de trasparencia de la Universidad Nacional.

Por último, respecto al presupuesto asignado para la ejecución de los programas, proyectos y actividades académicas (PPAA), la estructura de formulación presupuestaria de la Universidad Nacional no permite identificar los recursos operativos por quehacer sustantivo. Adicionalmente, el sistema de formulación de proyectos no registra los presupuestos asignados por las distintas instancias a los PPAA. Por lo tanto, en este momento no es posible identificar con certeza los montos o porcentaje del presupuesto universitario destinado a la extensión.

\section{Discusión}

En los últimos años la Universidad Nacional ha registrado un aumento en el total de matrícula, pasando de 14266 a 19393 estudiantes. La figura 1 muestra el crecimiento constante en esos datos. El incremento en la cantidad de estudiantes matriculados demuestra una labor sostenida de la Universidad en el ámbito de la docencia, entendida como oferta de educación formal. 
Por su parte, la figura 2 permite apreciar el desarrollo desigual de los proyectos según el quehacer sustantivo, como se puede apreciar la investigación, a pesar del decrecimiento experimentado en los últimos tres años, supera en más de tres veces el número de proyectos de extensión. Así por ejemplo en el año 2016 los proyectos de investigación representaron el 34\% los PPAA ejecutados, mientras que la extensión solo un $10 \%$ del total.

Contando como respaldo con la información cuantitativa que justifica este trabajo y considerando los datos cuantitativos recuperados, se propone que las instancias académicas de la Universidad Nacional formulen un programa académico, responsable de articular e integrar la docencia, la investigación y la extensión universitarias, de tal manera que los proyectos expresen la acción articulada de los quehaceres sustantivos en la relación universidad-sociedad, y se compartan los ejes estratégicos a desarrollar.

Con base en lo expuesto por Salom (2015), se entiende que el programa académico es un instrumento de planificación y ejecución de una propuesta académica que va más allá del planteamiento del trabajo fragmentado; por el contrario, lo que pretende es una verdadera articulación de la labor universitaria. De tal modo que se logre una armonización entre la oferta académica, las labores de investigación y las prácticas de extensión, formando un todo dinámico que cuente con espacios de encuentro y retroalimentación, así una se nutre de la otra en una relación simbiótica de beneficios para el gran concepto que se desea lograr de academia. La figura 3 pretende ilustrar dicha articulación.

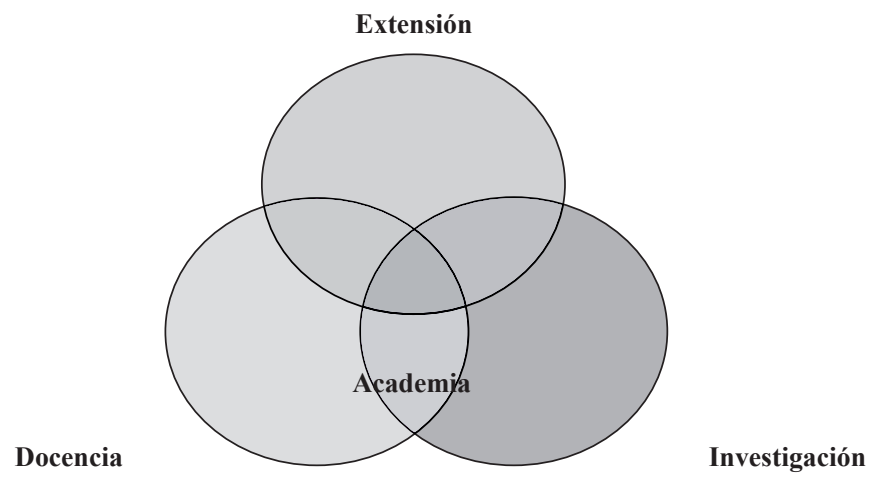

Figura 3. Integración académica

Fuente: elaboración propia 
Por lo anterior, como punto de partida, las instancias universitarias deben formular una misión que responda a los objetivos propios disciplinarios y a la misión de la Universidad. Toda acción debe atender a los principios, valores y fines de la Universidad Nacional.

Además, el programa académico contendría los PPAA, y correspondería a los académicos y las académicas la formulación, ejecución y evaluación de los mismos. Con lo cual se promueve una visión que responda a un concepto integrado de universidad, consciente de las realidades nacionales y regionales, $\mathrm{y}$ atenta a contribuir en las transformaciones necesarias.

El programa académico debe atender a una formulación de mediano plazo, en periodos no mayores a los cinco años, al tiempo que debe estar abierto a las evaluaciones y modificaciones que pueda requerir.

La Universidad tendrá que garantizar los recursos necesarios para su puesta en práctica mediante los fondos ordinarios destinados a cada instancia, sin que esto excluya la posibilidad de captar recursos por medio del vínculo externo remunerado. Por otra parte, las instancias administrativas deberán brindar el soporte necesario. Respecto a las jornadas académicas para su desarrollo, se considera como punto de partida la propuesta planteada por el Mag. Roy Atencio Morales, académico de la Universidad Nacional, la cual establece que quien sea nombrado con jornada académica debe destinar una de cada diez horas a la investigación y extensión, es decir, un $10 \%$ de la jornada. Según el académico, la investigación y la extensión deben formar parte de los criterios de distribución de jornadas, y considera la posibilidad de sustituir el tiempo destinado a la sistematización y producción didáctica con el propósito de cumplir con dicha propuesta.

Por otra parte, el programa académico debe proponerse incidir puntualmente y como modelo en las variables que determinan la calidad de vida de la sociedad. No se deben realizar programas, proyectos y actividades que sustituyan la labor del Estado, además, conviene no crear dependencia, por tanto, tienen que tener un carácter transitorio.

Por último, todas las acciones a emprender deben tener una finalidad académica, es decir, procurar contribuir al diálogo de saberes y considerar el abordaje de las capacidades. El desarrollo dialógico de capacidades y de potencialidades de las personas, de manera individual y/o grupal. 


\section{Conclusiones}

La política de extensión 2018-2028 debe contemplar como una de sus acciones promover en las instancias académicas la formulación de un programa académico, responsable de articular e integrar la docencia, la investigación y la extensión universitarias, de tal manera que los proyectos expresen la acción articulada de los quehaceres sustantivos en la relación universidad-sociedad, y se compartan los ejes estratégicos a desarrollar.

Dentro de las metas debe estar lograr sensibilizar y concientizar a la comunidad universitaria sobre la relevancia de la extensión como una función sustantiva de la universidad.

Además, se debe procurar la distribución de jornadas académicas, con criterios de equidad y proporcionalidad para la extensión, para la investigación y para la docencia. Cada persona que sea nombrada con jornada académica deberá destinar, como mínimo, un $10 \%$ a la realización de investigación y extensión.

La extensión universitaria es la forma más clara en que podemos dar a conocer nuestra labor a la sociedad y, lo más importante, en la práctica incorpora la participación ciudadana como un actor relevante en todas las fases del programa, proyecto o actividad.

Debemos por tanto recuperar la importancia de la extensión como actividad académica diferenciadora tanto de la Universidad Nacional como de las universidades públicas en general, en contraposición con propuestas que procuran limitar el quehacer universitario. 


\section{Referencias bibliográficas}

Atencio, R. (2017). El quehacer universitario. Universidad Nacional. Entrevista. Campus Coto. Corredores.

Salom, R. (2015). Acción social en la Universidad de Costa Rica. Ponencia presentada en el Primer Foro Institucional 2015: La Universidad de Costa Rica: aportes para el fortalecimiento del Estado Social de Derecho, la Institucionalidad Democrática y las Garantías Sociales. San Pedro. Costa Rica.

Universidad Nacional de Costa Rica. (2017a). Plan de mediano plazo (20132017). Recuperado de http://www.transparencia.una.ac.cr/index.php/ categorias/organizacion/planificacion

Universidad Nacional de Costa Rica. (2017b). Plan de mediano plazo institucional (2017-2021). Recuperado de http://www.transparencia. una.ac.cr/index.php/categorias/organizacion/planificacion

Universidad Nacional de Costa Rica. (2017c). UNA en cifras: educación superior para el desarrollo del país. Recuperado de http://www.transparencia.una.ac.cr/index.php/categorias/ una-en-cifras/376-educacion-superior-para-el-desarrollo-del-pais 
